\title{
Efeito da densidade de alojamento sobre a incidência de pododermatite e características ósseas de frangos de corte criados em aviários Dark House
}

\author{
Effect of housing density on pododermatitis incidence and bone characteristics in \\ broilers reared in "Dark House" system
}

\section{CRISTO, Adrieli Braga de ${ }^{1 *}$; SCHIMIDT, Joice Meri ${ }^{1}$; PERINI, Rafaela ${ }^{2}$; MORA, Mateus $^{2}$; MARQUES, Patrícia Fernanda dos Santos ${ }^{2}$; SANTOS, Alexandre Lesseur $\operatorname{dos}^{2}$; FERNANDES, Jovanir Inês Muller ${ }^{2}$}

\author{
${ }^{1}$ Universidade Federal do Paraná, Programa de Pós-Graduação em Ciência Animal, Departamento de \\ Zootecnia, Palotina, Paraná, Brasil. \\ ${ }^{2}$ Agroindústria Avícola, Palotina, Paraná, Brasil \\ *Endereço para correspondência: adrielibcristo@gmail.com
}

\section{RESUMO}

O objetivo foi avaliar o efeito da densidade de alojamento sobre a incidência de pododermatite e características ósseas durante todo período de criação de frangos de corte. $\mathrm{O}$ experimento foi realizado em três aviários comerciais. Os pintos foram alojados no mesmo dia e distribuídos de acordo com três densidades: aviário $\mathrm{A}-14.33$ aves $/ \mathrm{m}^{2}$, aviário $\mathrm{B}-15.00$ aves $/ \mathrm{m}^{2}$ e aviário $\mathrm{C}-$ 15.66 aves $/ \mathrm{m}^{2}$. Semanalmente 15 aves/aviário foram sacrificadas e as pernas e o pé direito de cada ave foram coletados. Foi avaliado o score macroscópico da área do coxim plantar de cada ave, caracterizando-os em: ausência de lesão (score A), lesão leve apenas no coxim plantar (score B), lesões graves e múltiplas (C). Em seguida, foi extraído o coxim plantar do pé e fixado em formol tamponado. As amostras foram submetidas aos procedimentos histológicos (medida da derme e epiderme). Os ossos das pernas foram submetidos ao ensaio de flexão à taxa de deformação. A imagem transversal da porção medial da diáfíse óssea foi capturada e medidas a área, espessura e diâmetro do osso cortical. Os dados foram analisados pelo programa SAS. O aumento na densidade de alojamento resultou em maior incidência de lesões graves no coxim plantar, menor resistência óssea à quebra e área do corte longitudinal da tíbia aos 42 dias e diminuição da espessura do osso cortical aos 21 dias de idade das aves. O aumento da densidade de alojamento afeta negativamente as características ósseas e leva a maior incidência de condenações por pododermatite ao abate.

Palavras-chave: calo de pé, inflamação, resistência óssea, umidade da cama

\section{SUMMARY}

The aim of this study was to evaluate the effect of housing density on the incidence of foot pad dermatitis and bone characteristics throughout rearing of broiler chickens. The experiment was carried out in three commercial poultry farms. The chicks were housed on the same day and distributed according to three densities: barn A 14.33 birds $/ \mathrm{m}^{2}$, barn $\mathrm{B}-15: 00$ birds $/ \mathrm{m}^{2}$ and barn C -15.66 birds / $\mathrm{m}^{2} .15$ birds / barn were weekly culled; legs and the right foot of each bird were collected. It was evaluated the macroscopic score of cushion plant area of each bird, classifying them into: no lesion (score A), bruises in the footpad (score B), serious and multiple injury (C). Then, it was extracted the footpad of the foot and fixed in formalin. The samples underwent histological procedures. The leg bones were tested for flexural deformation rate. The cross-sectional image of the medial portion of the bone was captured; then, the area, thickness and diameter were measured at the cortical bone. Data were analyzed through SAS software. The increased density resulted in higher incidence of severe lesions in the footpad, lower bone breaking strength and area 
of the cross section of the tibia after 42 days. It decreases the thickness of the cortical bone at 21 days of age of the birds. Increased housing density negatively affects bone characteristics and leads to higher incidence of food pad dermatitis at slaughter.

Keywords: foot callus, inflammation, bone strength, litter moisture

\section{INTRODUÇÃO}

A produção atual de frangos de corte no Brasil está em constante ascensão, o país se destaca como grande potencia mundial em produção de carne de frango. Com produção de 13,136 milhões em 2015, volume $3,5 \%$ superior ao obtido no ano anterior, o Brasil assume o segundo lugar no ranking dos maiores produtores de carne de frango, superando a China (TURRA, 2016).

Dentre os cortes de frango comercializados, os pés têm alto valor no mercado externo, principalmente $\mathrm{o}$ asiático os quais consideram esses cortes como iguarias. A exportação de pés de frango representa o terceiro produto mais importante economicamente para o país, depois de peito e asas (SHEPHERD \& FAIRCHILD, 2010). O maior fator limitante da qualidade dos pés de frango de corte é a pododermatite, lesão que se caracteriza pela inflamação do coxim plantar das aves, com consequente depreciação ou condenações no abatedouro e prejuízos econômicos para a indústria e o produtor e que claramente compromete o bem estar das aves (SANTOS et al., 2002). A ocorrência de pododermatite é utilizada como um critério de auditoria nas avaliações de bem-estar dos sistemas de produção de aves na Europa e nos Estados Unidos (NCC, 2010).

Diversas empresas têm aumentado a densidade de alojamento com o objetivo de maximizar a produção e diminuir os custos na criação de frangos de corte. No entanto, essa prática está associada ao aparecimento das lesões de pododermatite (BILGILI et al., 2006). Alguns estudos têm relatado que densidades de alojamento mais elevadas de aproximadamente 17.00 aves $/ \mathrm{m}^{2}$ estão associadas a maior incidência de pododermatite quando comparadas a densidades de alojamento inferiores de 8.00 aves $/ \mathrm{m}^{2}$ (MELUZZI et al., 2008). Buijs et al. (2009) verificaram que a pododermatite foi afetado negativamente quando a densidade alcançou 21.80 aves $/ \mathrm{m}^{2}$. O aumento na densidade de alojamento pode gerar uma piora no desempenho e redução na qualidade da cama principalmente por compactação, devido ao aumento de umidade, acarretando em lesões inflamatórias no coxim plantar das aves (GOPINGER et al, 2015).

Estudos relacionados a fragilidade óssea também têm demostrando haver correlação negativa entre aumento de densidade de alojamento e qualidade óssea (KUN et al., 2009; MENDES et al., 2012).

Devido ao alto valor agregado deste produto e crescentes problemas ligados ao bem-estar, é de grande interesse da indústria avícola desenvolver estratégias capazes de manter a qualidade de pés de frango, melhorar a qualidade óssea e diminuir as condenações do produto final (SHEPHERD \& FAIRCHILD, 2010). O objetivo deste trabalho foi avaliar o efeito da densidade de alojamento sobre a incidência de pododermatite e as características ósseas em frangos de corte criados em aviários Dark House.

\section{MATERIAL E MÉTODOS}

O experimento foi realizado no período de julho a agosto, em três aviários 
comerciais $\left(1,500 \quad \mathrm{~m}^{2}\right)$ de uma agroindústria local, com tecnologia similar (sistema Dark House). As camas eram compostas de maravalha e possuíam o mesmo tempo de uso ( $10^{\mathrm{o}}$ lote). Os pintos, procedentes do mesmo núcleo de matrizes de 34 semanas de idade, foram alojados no mesmo dia e distribuídos de acordo com três densidades: aviário $\mathrm{A}$ 14.33 aves $/ \mathrm{m}^{2}$ (21.500 aves), aviário B 15.00 aves $/ \mathrm{m}^{2}$ (22.500 aves) e aviário $\mathrm{C}$ 15.66 aves $/ \mathrm{m}^{2} \quad(23.500$ aves $)$ O experimento foi desenvolvido após aprovação pelo Comitê de Ética no Uso de Animais da Universidade Federal do Paraná sob protocolo n.051/2014.

Semanalmente, durante todo o período experimental (1 a 42 dias de idade), foram realizadas avaliações macroscópicas do coxim plantar das aves nos três aviários em estudo. Foram escolhidos seis pontos no aviário, sendo avaliadas 15 aves em cada ponto, totalizando 120 aves/aviário. Após essa mensuração, 15 aves/aviário (45 aves/ semana) foram sacrificadas e as pernas e os pés direito de cada ave foram coletados. Foi avaliado o score macroscópico da área do coxim plantar de cada ave caracterizando-os da seguinte forma: ausência de lesão (score A), lesão leve apenas no coxim plantar (score B), lesões graves e múltiplas $(\mathrm{C})$.

$\mathrm{O}$ coxim plantar foi fixado em formol tamponado. As amostras foram submetidas aos procedimentos histológicos e coradas por hematoxilinaeosina para análise morfométrica (medida da derme e epiderme). As imagens foram capturadas e a leitura foi realizada através do analisador de imagem computadorizado Image Proplus 5.2, da Mídia Cibertecnics. Foram realizadas 10 medidas da derme e epiderme de cada coxim plantar e calculada a média.

Após remoção de todo tecido aderente da perna direita, a tíbia foi submetida ao ensaio de flexão à taxa de deformação constante para material visco-elástico, com equipamento de ensaio universal da Marca Texture Analyser, com célula de carga de $500 \mathrm{kgf}$, velocidade do cabeçote de $10 \mathrm{~mm} / \mathrm{seg}$. A imagem transversal da porção medial da diáfise óssea foi fotografada e capturada para medida da área, espessura e diâmetro do osso cortical. Para a leitura foi utilizado o analisador de imagem computadorizado IMAGE PROPLUS 5.2, da Mídia Cibertecnics.

Os resultados obtidos foram tabulados e analisados utilizando-se análise de variância do procedimento General Lineal Model (GLM) com auxílio do programa estatístico SAS (2002, SAS Institute Inc., Cary, NC) e quando significativas, as médias entre os tratamentos foram comparadas pelos testes de Tukey a 5\%. Para os dados que não se comportaram na distribuição normal pelo teste de KruskalWallis foram transformados de acordo com o perfil da variável e/ou realizado análises não paramétricas.

\section{RESULTADOS E DISCUSSÃO}

Até os 28 dias de idade não houve diferença significativa $(\mathrm{P}>0,05)$ para as medidas de derme e epiderme em aves criadas em diferentes densidades (Tabela 1). Aos 35 dias, é possível observar maior espessura de derme e epiderme em aves alojadas em densidades intermediárias, indicando um possível processo inflamatório no coxim plantar dessas aves. Estes resultados contrariam os encontrados por Mendes et al. (2012), os quais mostram que altas densidades de alojamento afetam a intensidade de problemas locomotores e de lesões no coxim plantar das aves, aumentando gradativamente com o desenvolvimento das mesmas.

Os resultados do presente estudo podem ser explicados por alguma falha no 
manejo da cama das aves alojadas em densidade intermediaria. Uma vez que o material da cama era similar para todos os aviários estudados, falhas no manejo da cama podem permitir o crescimento microbiano e a proliferação de doenças. A alta volatilização da amônia é um reflexo das condições de manejo inadequadas da cama, o que pode levar ao comprometimento do bem-estar e ao aumento da incidência de pododermatites de contato (NAGARAJ et al., 2007).

Tabela 1. Medidas da espessura de derme e epiderme $(\mathrm{mm})$ de frangos de corte de 7 a 42 dias de idade criados em três aviários Dark House de diferentes densidades

\begin{tabular}{lcccccc}
\hline Item & 7 dias & 14 dias & 21 dias & 28 dias & 35 dias & 42 dias \\
\hline 14,33 aves $/ \mathrm{m}^{2}$ & 186,67 & 186,051 & 241 & 303,74 & $320,86^{\mathrm{b}}$ & 554,35 \\
15,00 aves $/ \mathrm{m}^{2}$ & 182,93 & 157,92 & 246,29 & 302,02 & $466,44^{\mathrm{a}}$ & 446,50 \\
15,66 aves $/ \mathrm{m}^{2}$ & 177,09 & 175,31 & 248,24 & 338,03 & $302,87^{\mathrm{b}}$ & 497,41 \\
$\mathrm{CV}, \%$ & 16,79 & 22,89 & 21,50 & 24,01 & 42,60 & 32,47 \\
Valor de P & 0,7402 & 0,2445 & 0,9412 & 0,4634 & 0,0323 & 0,3865 \\
\hline
\end{tabular}

Médias seguidas de letras distintas nas colunas diferem entre si pelo teste de Tukey.

Não houve diferença significativa $(\mathrm{P}<0,05)$ para as avaliações microscópicas das lesões, exceto para inflamação crônica grave (Tabela 2). Aves criadas em densidades intermediarias apresentaram maior incidência, indicando um processo inflamatório de longa evolução.

O aparecimento da lesão, que inicia com uma inflamação da pele, em geral está associado a fatores corrosivos presentes na cama e que pode evoluir na presença de Staphylococcus ssp. (DUMAS et al.,
2011). Dozier et al. (2005) relataram que o aumento de lesões devido a altas densidades e o crescimento das aves, é provavelmente um reflexo da baixa qualidade da cama. Portanto, o manejo inadequado da cama desde o início do período de criação das aves, que permitam o acúmulo de umidade $\mathrm{e}$ amônia e compactação da mesma, favorecem o aparecimento e agravamento das lesões inflamatórias dos coxins plantar das aves.

Tabela 2. Avaliações microscópicas de coxim plantar de frangos de corte criados em três aviários Dark House de diferentes densidades

\begin{tabular}{lcccccc}
\hline Aves $/ \mathrm{m}^{2}$ & Ulcera & Erosão & $\begin{array}{c}\text { Inflamação } \\
\text { aguda leve }\end{array}$ & $\begin{array}{c}\text { Inflamação } \\
\text { aguda grave }\end{array}$ & $\begin{array}{c}\text { Inflamação } \\
\text { crônica leve }\end{array}$ & $\begin{array}{c}\text { Inflamação } \\
\text { crônica grave }\end{array}$ \\
\hline 14,33 & 47,928 & 5,104 & 45,367 & 20,707 & 40,076 & $16,05^{\text {ab }}$ \\
15,00 & 48,926 & 1,038 & 20,765 & 17,210 & 23,000 & $34,17^{\text {a }}$ \\
15,66 & 52,958 & 4,643 & 28,896 & 17,848 & 32,512 & $16,02^{\mathrm{b}}$ \\
\hline
\end{tabular}

Médias seguidas de letras distintas nas colunas diferem entre si pelo teste de Kruskal-wallis.

O percentual de lesões graves (score C) no coxim plantar foi maior conforme houve aumento na densidade de criação durante toda a fase de criação (Tabela 3). Resultados semelhantes foram encontrados por Gospinger et al. (2015), 
onde aumento da densidade populacional de 11,08 para 13,20 aves $\mathrm{m}^{2}$ levou a uma maior ocorrência de lesões de pododermatite de score $\mathrm{C}$. $\mathrm{O}$ aumento populacional de aves por $\mathrm{m}^{2}$ nos aviários resulta em maior liberação de excretas e consequentemente eleva a umidade na cama (LOPES et al., 2012), predispondo a ocorrência de pododermatite (MAYNE et al., 2007).

Souza \& Molento (2015) estudaram os programas de boas práticas agropecuárias (BPA) de 15 empresas exportadoras de frangos de corte do Paraná, responsáveis por $76,3 \%$ da produção do estado. Esse estudo apontou que $93,3 \%$ dessas companhias implementaram o sistema Dark House e mais da metade utilizam cinco luxes de intensidade luminosa. Nessas condições, a pododermatite foi a doença mais citada como indicador sanitário $(93,3 \%)$. A baixa intensidade luminosa, segundo os autores do estudo, pode diminuir a atividade e ainda contribuir com a incidência de dermatites de contato e desordens locomotoras.

Tabela 3. Comportamento do percentual de scores de lesões de pododermatite de frangos de corte de 7 a 42 dias de idade criados em três aviários Dark House de diferentes densidades

\begin{tabular}{lccccccc}
\hline \multirow{2}{*}{ Item } & 7 dias & 14 dias & 21 dias & 28 dias & 35 dias & 42 dias & Efeito \\
\cline { 2 - 8 } & \multicolumn{7}{c}{ Score A } \\
\hline 14,33 aves $/ \mathrm{m}^{2}$ & 48,33 & 24,17 & 22,50 & 20,00 & 23,33 & 21,67 & Quadrático \\
15,00 aves $/ \mathrm{m}^{2}$ & 37,50 & 22,50 & 10,00 & 18,33 & 12,50 & 17,50 & Quadrático \\
15,66 aves $/ \mathrm{m}^{2}$ & 23,33 & 19,17 & 11,67 & 6,67 & 8,33 & 6,67 & Quadrático \\
\hline \multicolumn{7}{c}{ Score B } \\
\hline 14,33 aves $/ \mathrm{m}^{2}$ & 33,33 & 35,00 & 29,17 & 43,33 & 47,50 & 52,50 & Linear \\
15,00 aves $/ \mathrm{m}^{2}$ & 35,83 & 49,17 & 33,33 & 44,17 & 52,50 & 39,17 & NS \\
15,66 aves $/ \mathrm{m}^{2}$ & 30,00 & 44,17 & 31,67 & 39,17 & 45,00 & 45,83 & NS \\
\hline \multicolumn{7}{c}{ Score C } \\
\hline 14,33 aves $/ \mathrm{m}^{2}$ & 18,33 & 40,83 & 48,33 & 36,67 & 29,17 & 25,83 & Quadrático \\
15,00 aves $/ \mathrm{m}^{2}$ & 26,67 & 28,33 & 56,67 & 37,50 & 35,00 & 43,33 & Linear \\
15,66 aves $/ \mathrm{m}^{2}$ & 46,67 & 36,67 & 56,67 & 54,17 & 46,67 & 47,50 & NS \\
\hline
\end{tabular}

Score $\mathrm{A}=$ ausência de lesão, Score $\mathrm{B}=$ - lesão leve apenas no coxim plantar e Score $\mathrm{C}=$ - lesões graves $\mathrm{e}$ múltiplas.

Os scores A, B e C para densidade de 14,33 aves $/ \mathrm{m}^{2}$ há efeito linear para as lesões de grau leve (score B), conforme aumenta a idade do lote. Para lesões graves (score C) e ausência de lesões (score A) houve efeito quadrático. Esses resultados são devidos, provavelmente, à aleatoriedade durante as coletas nas diferentes idades e à variação individual que esses parâmetros apresentam.

Quanto à ausência (score A), grau leve (score B) ou grave (score C) de lesões para densidade de 15,00 aves $/ \mathrm{m}^{2}$ houve aumento das lesões graves de forma linear até os 42 dias de idade. Os resultados do presente estudo estão de acordo com os encontrados por Muniz et al. (2006), mostrando haver uma relação direta entre densidade por metro quadrado e a percentagem de lesões de pés devido ao volume de excretas eliminado, interferindo negativamente na saúde e bem-estar das aves. 
Conforme aumenta a densidade de criação, há a redução da ausência de lesões e o aumento no percentual $(\mathrm{P}<0,05)$ de lesões graves no coxim plantar das aves de 1 a 42 dias de idade e ao abate (Tabela 4). Oliveira, et al. (2002) estudaram a influência da densidade sobe lesões de pododermatite e também relataram maior incidência de lesões graves conforme houve o aumento da densidade e a piora na qualidade de cama, principalmente devido ao acúmulo de umidade. Em destaque o percentual de aves com lesão de score $\mathrm{C}$ no abate, de $32,42 \%$ para as aves criadas na densidade mais baixa $\left(14,33\right.$ aves $\left./ \mathrm{m}^{2}\right)$ e 57,50 e $57,33 \%$, para as densidades média e mais alta, respectivamente, comprovando os efeitos negativos da alta lotação em perdas econômicas no momento do abate, visto que a pododermatite é um problema crítico que pode comprometer as exportações de pés e pernas de frangos de corte (HASHIMOTO et al., 2013).

Tabela 4. Percentual de lesões de pododermatite observadas em frangos de corte de 1 a 42 dias de idade e ao abate, criados em três aviários Dark House de diferentes densidades

\begin{tabular}{lcccccc}
\hline \multirow{2}{*}{ Item } & \multicolumn{3}{c}{1 a 42 dias, \% } & \multicolumn{3}{c}{ Abate, \% } \\
\cline { 2 - 6 } & Score A & Score B & Score C & Score A & Score B & Score C \\
\hline 14,33 aves $/ \mathrm{m}^{2}$ & $26,67^{\mathrm{a}}$ & 40,14 & $33,19^{\mathrm{c}}$ & $24,68^{\mathrm{a}}$ & 42,28 & $33,03^{\mathrm{b}}$ \\
15,00 aves $/ \mathrm{m}^{2}$ & $19,72^{\mathrm{b}}$ & 42,36 & $37,92^{\mathrm{b}}$ & $9,42^{\mathrm{b}}$ & 33,08 & $57,50^{\mathrm{a}}$ \\
15,66 aves $/ \mathrm{m}^{2}$ & $12,64^{\mathrm{c}}$ & 39,31 & $48,06^{\mathrm{a}}$ & $6,67^{\mathrm{b}}$ & 36,00 & $57,33^{\mathrm{a}}$ \\
$\mathrm{CV}, \%$ & 51,16 & 30,27 & 38,67 & 43,23 & 32,65 & 30,50 \\
Valor de P & 0,0102 & 0,1356 & 0,0102 & 0,0233 & 0,2457 & 0,0332 \\
\hline
\end{tabular}

Médias seguidas de letras distintas na coluna diferem entre si pelo teste de Tukey.

O aumento das lesões graves é mais evidente ao final da fase de criação (42 dias). Este é um reflexo da redução da eficiência alimentar conforme o aumento da idade das aves aumentando a liberação de nitrogênio na cama. Miles et al.(2008) observaram que os teores de nitrogênio total e amônio $\left(\mathrm{NH}_{4}{ }^{+}\right)$em cama de frangos de corte aumentam representativamente conforme ocorre $\mathrm{o}$ aumento da idade das aves, sendo esse também um dos fatores predisponentes das lesões de pododermatite.

Aos sete dias de idades, é possível observar que aves alojadas em baixas densidades apresentaram menor incidência de lesões (Score A). Aves alojadas em densidade baixa também apresentaram maior incidência de lesões graves (Score C) (Tabela 5). Segundo Mendes et al. (2012), a incidência de lesões quando as aves são muito jovens, pode ser explicado pela fragilidade da derme dos pintainhos, que adquirem maior resistência com a idade e, com isso, diminuíram a incidência de lesões na idade intermediária do lote.

Estudando a interferência de diferentes densidades sobre lesões de pododermatite, esses mesmos autores observaram que aos 42 dias, altas densidades de alojamento levam a maior incidência de lesões de pododermatite graves. Ao contrário do que foi encontrando neste estudo, onde houve maior incidência de lesões graves (Score C) em aves alojadas em baixa e média densidade, isso pode ser relacionado com a qualidade da cama, as quais podem ter apresentado pior qualidade quando comparadas com a cama e alta densidade devido falhas no manejo refletindo diretamente no aparecimento de pododermatite. 
Tabela 5. Percentual de scores de lesões de pododermatite de frangos de corte de $7 \mathrm{a}$ 42 dias de idade criados em três aviários Dark House de diferentes densidades

\begin{tabular}{|c|c|c|c|}
\hline & Score A & Score B & Score C \\
\hline \multicolumn{4}{|c|}{7 dias } \\
\hline 14,33 aves $/ \mathrm{m}^{2}$ & $48,333^{\mathrm{a}}$ & 35,833 & $46,667^{\mathrm{a}}$ \\
\hline 15,00 aves $/ \mathrm{m}^{2}$ & $37,500^{\mathrm{ab}}$ & 33,333 & $26,667^{\mathrm{b}}$ \\
\hline 15,66 aves $/ \mathrm{m}^{2}$ & $23,333^{b}$ & 30,000 & $18,333^{b}$ \\
\hline $\mathrm{CV}, \%$ & 31,76 & 32,71 & 33,27 \\
\hline Valor de P & 0,0060 & 0,6524 & 0,0007 \\
\hline \multicolumn{4}{|c|}{14 dias } \\
\hline 14,33 aves $/ \mathrm{m}^{2}$ & 24,167 & 49,167 & 40,833 \\
\hline 15,00 aves $/ \mathrm{m}^{2}$ & 22,500 & 44,167 & 36,667 \\
\hline 15,66 aves $/ \mathrm{m}^{2}$ & 19,167 & 35,000 & 28,333 \\
\hline $\mathrm{CV}, \%$ & 44,48 & 20,39 & 36,87 \\
\hline Valor de P & 0,6720 & 0,0387 & 0,2686 \\
\hline \multicolumn{4}{|c|}{21 dias } \\
\hline 14,33 aves $/ \mathrm{m}^{2}$ & 22,500 & 33,333 & 51,000 \\
\hline 15,00 aves $/ \mathrm{m}^{2}$ & 11,667 & 31,667 & 50,000 \\
\hline 15,66 aves $/ \mathrm{m}^{2}$ & 10,000 & 29,167 & 48,333 \\
\hline $\mathrm{CV}, \%$ & 71,69 & 36,67 & 22,69 \\
\hline Valor de P & 0,1172 & 0,8215 & 0,9244 \\
\hline \multicolumn{4}{|c|}{28 dias } \\
\hline 14,33 aves $/ \mathrm{m}^{2}$ & 20,000 & 42,500 & 54,167 \\
\hline 15,00 aves $/ \mathrm{m}^{2}$ & 18,333 & 42,000 & 37,500 \\
\hline 15,66 aves $/ \mathrm{m}^{2}$ & 6,667 & 39,167 & 36,667 \\
\hline $\mathrm{CV}, \%$ & 62,46 & 29,21 & 13,65 \\
\hline Valor de P & 0,0526 & 0,0479 & 0,5933 \\
\hline \multicolumn{4}{|c|}{35 dias } \\
\hline 14,33 aves $/ \mathrm{m}^{2}$ & $23,333^{\mathrm{a}}$ & 52,500 & 46,667 \\
\hline 15,00 aves $/ \mathrm{m}^{2}$ & $12,500^{\mathrm{ab}}$ & 47,500 & 35,000 \\
\hline 15,66 aves $/ \mathrm{m}^{2}$ & $8,333^{\mathrm{b}}$ & 45,000 & 29,167 \\
\hline $\mathrm{CV}, \%$ & 56,94 & 23,43 & 39,56 \\
\hline Valor de P & 0,0202 & 0,5207 & 0,1419 \\
\hline \multicolumn{4}{|c|}{42 dias } \\
\hline 14,33 aves $/ \mathrm{m}^{2}$ & $21,667^{\mathrm{a}}$ & 52,500 & $45,833^{\mathrm{a}}$ \\
\hline 15,00 aves $/ \mathrm{m}^{2}$ & $17,500^{\mathrm{a}}$ & 45,833 & $43,333^{a}$ \\
\hline 15,66 aves $/ \mathrm{m}^{2}$ & $6,667^{\mathrm{b}}$ & 39,167 & $25,833^{b}$ \\
\hline $\mathrm{CV}, \%$ & 46,41 & 34,86 & 28,10 \\
\hline Valor de $\mathrm{P}$ & 0,0066 & 0,3761 & 0,0086 \\
\hline
\end{tabular}

Médias seguidas de letras distintas nas colunas diferem entre si pelo teste de Tukey.

Feddes et al. (2002) constataram que o consumo de água foi maior em altas densidades de alojamento, o que resultou em excretas mais liquidas e consequentemente contribuiu para $\mathrm{o}$ aumento na umidade da cama. Meluzzi et al. (2008) avaliaram a relação da densidade de alojamento e as estações do ano, e observaram que a incidência de lesões foi significativamente maior em aves criadas no inverno em comparação com aqueles criadas no verão. Os autores relatam que a redução na ventilação com intuito de manter a temperatura torna-a insuficiente para remover o excesso de umidade do ar, o que pode explicar os resultados do presente estudo, que ocorreu nos meses de inverno. 
Portanto, além da densidade de alojamento, o manejo da ambiência no interior dos aviários pode contribuir negativamente com a gravidade das lesões de pododermatite (DAWKINS et al., 2004).

Para a resistência óssea, não houve diferença significativa $(\mathrm{P}>0,05)$ entre diferentes densidades de alojamento
(Tabela 6). Esses resultados estão de acordo com os encontrados por Oliveira et al. (2014). Por outro lado, Buijis et al. (2012) mostaram que o aumento da densidade levou a uma diminuição da resistência a quebra da tíbia, sugerindo um efeito negativo do aumento da densidade principalmente no bem-estar.

Tabela 6. Resistência óssea à quebra $(\mathrm{Kg})$ de frangos de corte criados em três aviários Dark House de diferentes densidades

\begin{tabular}{lccccccc}
\hline & 7 dias & 14 dias & 21 dias & 28 dias & 35 dias & 42 dias & Efeito \\
\hline 14,33 aves $/ \mathrm{m}^{2}$ & 2,82 & 7,76 & 18,42 & 23,92 & 39,82 & 45,12 & Linear \\
15,00 aves $/ \mathrm{m}^{2}$ & 3,05 & 7,86 & 16,42 & 23,94 & 37,18 & 29,18 & Linear \\
15,66 aves $/ \mathrm{m}^{2}$ & 2,95 & 7,89 & 17,44 & 23,47 & 38,46 & 38,24 & Linear \\
CV, \% & 15,04 & 16,79 & 18,23 & 18,23 & 19,60 & 22,66 & \\
Valor de $\mathrm{P}$ & 0,4087 & 0,9601 & 0,2311 & 0,9400 & 0,6511 & 0,0803 & \\
\hline
\end{tabular}

Aves alojadas em menor densidade apresentaram maiores valores de resistência óssea à quebra (Figura 1). Aves que realizam maior atividade física tendem a apresentar o osso mais resistente, e a maior atividade física seria proporcionada pelo maior espaço físico para movimentação das aves dentro do aviário (OLIVEIRA et al., 2014). Conforme Kun et al. (2009) o aumento da densidade de alojamento causa diminuição da qualidade óssea, com consequente aumento na incidência de fraturas ósseas e articulares.

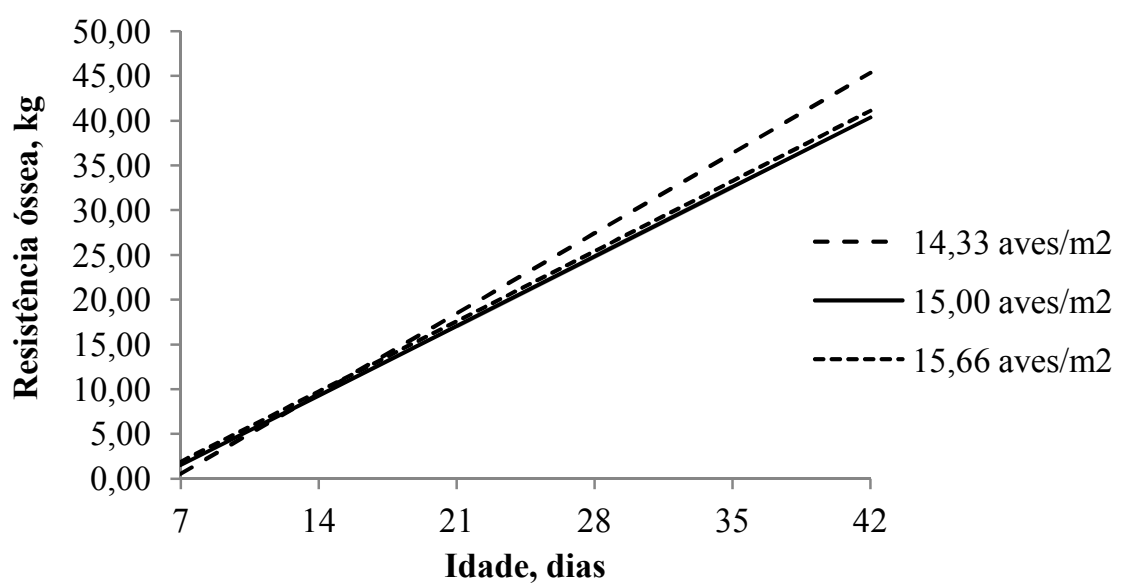

Figura 1. Resistência óssea à quebra de frangos de corte de 7 a 42 dias de idade criados em três aviários Dark House de diferentes densidades, Densidade de 14,33 aves $/ \mathrm{m}^{2}:(\hat{\mathrm{Y}}=-$ $\left.8,3914+1,2793 x ; \quad R^{2}=0,8707\right)$, densidade de 15,00 aves $/ \mathrm{m}^{2}:\left(\hat{\mathrm{Y}}=-6,2353+1,1097 \mathrm{x} ; \mathrm{R}^{2}=0,8539\right)$, densidade de 15,66 aves $/ \mathrm{m}^{2}:\left(\hat{\mathrm{Y}}=-5,9677+1,12035 \mathrm{x} ; \mathrm{R}^{2}=0,8593\right)$ 
A resistência à quebra mostrou-se crescente até aos 42 dias de idade, indicando que os ossos ainda se encontram em fase de formação neste período de criação. Esses resultados estão de acordo com por Bruno et al. (2007) que também encontraram correlação positiva nos quais a resistência óssea aumentou de acordo com a idade da ave.

A área do corte transversal da tíbia foi maior para as aves aos 42 dias criadas em menor densidade, quando comparadas com as aves criadas em maior densidade (Tabela 7). Mostrando que ocorre uma correlação positiva entre menores densidades e melhora da qualidade óssea. Esse fato pode ser explicado de uma ave que realiza maior atividade física teria um osso mais resistente, e a maior atividade física seria proporcionada pelo maior espaço físico para movimentação das aves (OLIVEIRA et al., 2014).

É possível observar que área do corte transversal da tíbia mostrou-se crescente, atingindo seu pico aos 42 dias de idade, indicando que o osso ainda encontra-se em fase de formação nesta idade.

Tabela 7. Área do corte transversal da tíbia $\left(\mathrm{mm}^{2}\right)$ de frangos de corte criados em três aviários Dark House diferentes densidades

\begin{tabular}{lccccccc}
\hline Item & 7 dias & 14 dias & 21 dias & 28 dias & 35 dias & 42 dias & Efeito \\
\hline 14,33 aves $/ \mathrm{m}^{2}$ & 4,48 & 7,05 & 12,30 & 17,82 & 24,79 & $30,17^{\text {a }}$ & Linear \\
15,00 aves $/ \mathrm{m}^{2}$ & 4,85 & 7,65 & 12,98 & 19,22 & 22,20 & $27,38^{\text {b }}$ & Linear \\
15,66 aves $/ \mathrm{m}^{2}$ & 4,76 & 7,58 & 10,72 & 19,37 & 24,07 & $25,72^{\mathrm{b}}$ & Linear \\
$\mathrm{CV}, \%$ & 17,08 & 25,44 & 29,63 & 23,03 & 16,70 & 18,37 & \\
Valor de P & 0,4602 & 0,77 & 0,21 & 0,59 & 0,24 & 0,056 & \\
\hline
\end{tabular}

Médias seguidas de letras distintas nas colunas diferem entre si pelo teste de Tukey.

Aves criadas em menores densidades apresentaram maior espessura do osso cortical aos 21 dias de idade (Tabela 8), corroborando com os resultados de maior resistência óssea em menores densidades de alojamento.

Mendes et al. (2012), ao avaliarem o efeito de altas densidades de alojamento sobre os problemas locomotores em frangos de corte, verificaram maiores índices nas densidades de 15 e 18 aves $/ \mathrm{m}^{2}$, o que indica resultados negativos na qualidade óssea de aves alojadas em altas densidades. A formação óssea adequada é fundamental, pois o desenvolvimento muscular e o desempenho zootécnico das aves são totalmente dependentes de um bom suporte ósseo.

Tabela 8. Espessura do osso cortical de frangos de corte de 7 a 42 dias de idade, criados em três aviários Dark House de diferentes densidades

\begin{tabular}{lccccccc}
\hline Densidade & 7 dias & 14 dias & 21 dias & 28 dias & 35 dias & 42 dias & Efeito \\
\hline 14,33 aves $/ \mathrm{m}^{2}$ & 0,68 & 0,82 & $1,12^{\mathrm{a}}$ & 1,30 & 1,35 & 1,54 & Linear \\
15,00 aves $/ \mathrm{m}^{2}$ & 0,72 & 0,84 & $1,17^{\mathrm{a}}$ & 1,30 & 1,26 & 1,90 & Linear \\
15,66 aves $/ \mathrm{m}^{2}$ & 0,74 & 0,90 & $0,87^{\mathrm{b}}$ & 1,39 & 1,30 & 1,56 & Linear \\
$\mathrm{CV}, \%$ & 14,41 & 24,49 & 30,51 & 14,72 & 15,29 & 47,32 & - \\
Valor de P & 0,2912 & 0,6743 & 0,0211 & 0,4109 & 0,5723 & 0,3901 & - \\
\hline
\end{tabular}

Médias seguidas de letras distintas nas colunas diferem entre si pelo teste de Tukey. 
O diâmetro dos ossos não foi alterado $(\mathrm{P}>0,05)$ em nenhuma idade avaliada (Tabela 9). Segundo Webster (2004), a restrição da atividade e movimentação das aves criadas em gaiolas contribui para a maior fragilidade óssea tornandoas mais susceptíveis a fraturas dolorosas. Esse mesmo efeito pode ser percebido em frangos de corte criados em alta densidade visto que nestas condições, também há restrição de movimentos.

Os problemas que acometem o sistema locomotor de animais confinados são de grande importância econômica na produção animal, pois além de afetar diretamente o bem estar das aves, pode ainda resultar em queda no desempenho zootécnico (ALMEIDA et al., 2009).

Tabela 9. Diâmetro do osso cortical (mm) de frangos de corte de 7 a 42 dias de idade, criados em três aviários Dark House de diferentes densidades

\begin{tabular}{lllllll}
\hline Densidade & 7 dias & 14 dias & 21 dias & 28 dias & 35 dias & 42 dias \\
\hline 14,33 aves $/ \mathrm{m}^{2}$ & 2,65 & 3,18 & 4,40 & 5,57 & 6,79 & 7,57 \\
15,00 aves $/ \mathrm{m}^{2}$ & 2,76 & 3,39 & 4,56 & 5,70 & 6,54 & 7,21 \\
15,66 aves $/ \mathrm{m}^{2}$ & 2,66 & 3,39 & 4,49 & 5,62 & 6,61 & 7,28 \\
CV, \% & 7,31 & 11,15 & 9,29 & 13,91 & 10,09 & 16,87 \\
Valor de P & 0,2799 & 0,4103 & 0,6002 & 0,9066 & 0,6212 & 0,7245 \\
\hline
\end{tabular}

Embora haja compreensão dos fatores que afetam a incidência e a gravidade das lesões de pododermatite, essa condição ainda é um problema econômico e de bem estar e se torna ainda mais importante a medida a demanda por pés de qualidade aumenta pelos mercados exportadores. De acordo com Shepherd \& Fairchild (2010), o processo exato que resulta em pododematite não é claramente compreendido, embora a maioria das pesquisas concorda que a qualidade e o tipo da cama são importantes fatores predisponentes no aparecimento da pododermatite, o sobrepeso das atuais linhagens de frangos de corte $\mathrm{e}$ consequente contaminação por bactérias como Staphylococcus spp devem ser considerados. A nutrição pode influenciar diretamente a umidade da cama e precisa ser considerada como um fator relevante no desenvolvimento de lesões nos coxins plantares.

$\mathrm{O}$ aumento na densidade de alojamento resultou em maior incidência de lesões graves no coxim plantar, menor resistência óssea à quebra e área do corte longitudinal da tíbia aos 42 dias e diminuição da espessura do osso cortical aos 21 dias de idade das aves.

Sanotra et al. (2011) estudaram a influência de programas de luz e densidade de alojamento sobre o comportamento, risco de problemas de perna e ocorrência de medo crônico em frangos de corte, e então concluíram que aves criadas com programas de luz apresentaram menos problemas de perna e foram menos medrosas. Além disso, as aves conseguiram manter ritmo diurno e estabelecer padrões de comportamento como comer, dormir, descansar e se locomover de acordo com o dia e a noite. Entretanto, apesar das vantagens do sistema Dark House devido ao maior potencial para produção com desempenho superior aos sistemas convencionais de cortina amarela, o aumento da densidade de alojamento é um fator importante a ser considerado em relação à ocorrência de pododermatites. Conforme observado nesse estudo, o aumento da densidade de 
alojamento em aviários Dark House afeta negativamente as características ósseas e leva a maior incidência de condenações por pododermatite ao abate de frangos de corte

Segundo Souza \& Molento (2015), as informações a respeito da ocorrência de pododermatite em aviários Dark house é bastante inconsistente. Em alguns estudos compilados pelos autores, são referidas ocorrências de 3,0\% a 25,0\%, chegando a variação de $55,0 \%$ a $82,0 \%$. A falta de informações mais precisas das taxas máximas de ocorrência da pododermatite sugere uma dificuldade das companhias em desenvolver indicadores de avaliação, assim como de correlacionar os principais fatores envolvidos. Uma vez que injurias como a pododermatite e os problemas locomotores indicam uma situação de desconforto e que comprometem o bem estar das aves, é importante priorizar estudos que estabeleçam e melhorem os indicadores sanitários neste tipo de instalação.

\section{REFERENCIA}

ALMEIDA, I.C.L.P.; MENDES, A.A.; MARTINS, M.R.F.B.; FERNANDES, B.C.S.; ALMEIDA, I.C.L.; MILBRADT, E.L.; BALOG, A.; KOMIYAMA, C.M. Followup of the development of femoral degeneration lesions in broilers. International Journal of Morphology, v.27, n.2, p.571-575, 2009.

BIGILI, S.F.; ALLEY, M.A.; HESS, J.B.; NAGARAJ, M. Influence of age and sex on foot pad quality and yield in broiler chickens reared on low and high density diets. Journal of Applied Poultry Research, v.15, n.3, p.433441, 2006.
BRUNO, L.D.G.; LUQUETTI, B.C.; FURLAN, R.L.; MACARI, M.

Influence of early quantitative feed restriction and environmental temperature on long bone development of broiler chickens. Journal of Thermal Biology, v.32, n.6, p.349-354, 2007.

BUIJS, S.; POUCKE, E. V.; TUYTTENS, F.A.M.; KEELING, L.; RETTENBACHER, S. Stocking density effects on broiler welfare: Identifying sensitive ranges for different indicators. Poultry Science, v.88, n.8, p.15361543, 2009.

DAWKINS, M.S.; DONNELLY, C.A.; JONES, T.A. Chicken welfare is influenced more by housing conditions than by stocking density. Nature, v.427, n.6972, p.342-344, 2004.

DOZIER, W.A.; THAXTON, J.P.; BRANTON, S.L.; MORGAN, G.W.; MILES, D.M.; ROUSH, W.B.; LOTT, B.D. AND VIZZIER-THAXTON, Y. Stocking density effects on growth performance and processing yields of heavy broilers. Poultry Scince, v.84, n. 8, p.1332-1338, 2005.

DUMAS, M.D.; POLSON, S.W.; RITTER, D.; RAVEL, J.; GELB, J.JR. Impacts of Poultry House Environment on Poultry Litter Bacterial Community Composition. PLoS ONE, v.6, n.9, 2011. Doi: 0.1371/journal.pone.0024785.

FEDDES, J.J.; EMMANUEL, E.J.; ZUIDHOFT, M.J. Broiler performance. body weight variance, feed and water intake and carcass quality at different stocking densities. Poultry Science, v.81, n.6, p.774-779, 2002. 
GOPINGER, E.; AVILA, V.S.; PERONDI, D.; CATALAN, A.A.S.; KRABBE, E. L.; ROLL, V.F.B.

Performance, carcass characteristics and litter moisture in broilers housed at two densities. Acta Scientiarum. Animal Sciences, v.37, n.1, p.35-39, 2015.

HASHIMOTO, S.; YAMAZAKI, K.; OBI, T.; TAKASE, K. Relationship between severity of footpad dermatitis and carcass performance in broiler chickens. The Journal of Veterinary Medical Science, v.75, n.11, p.15471549, 2013.

KUN, Z.; ULUOCAK, A.N.; KARAMAN, M. The influence of some factors on carcass defects during fattening period in broilers. Archivos de

Zootecnia, v.58, n.221, p. 117-120, 2009.

LOPES, M.; PIRES, P.G.S.; ROLL, V.F.B.; VALENTE, B.S.; ANCIUTI, M.A. Pododermatite em aves.

Publicações em Medicina Veterinária e Zootecnia, v.6, n.32, p.1459, 2012.

MAYNE, R.K.; ELSE, R.W.; HOCKING, P.M. High litter moisture alone is sufficient to cause footpad dermatitis in growing turkeys. British Poultry Science, v.48, n.5, p.538-545, 2007.

MELUZZI, A.; FABBRI, C.; FOLEGATTI, E.; SIRRI, F. Survey of chicken rearing conditions in Italy: Effects of litter quality and stocking density on productivity. foot dermatitis and carcase injuries. British Poultry Science, v.49, n.3, p.257-264, 2008.

MENDES, A.S.; PAIXÃO, S.J.; MROSTEGA, J.B.; RESTELATTO, R.C.; OLIVEIRA, P.A.V.; POSSENTI, J.C. Mensuração de problemas locomotores e de lesões no coxim plantar em frangos de corte. Archivo de

Zootecnia, v.61, n.234, p.217-228, 2012.
MILES, D.M.; ROWE, D.E.; OWENS, P.R. Winter broiler litter gases and nitrogen compounds: temporal and spatial trends. Atmospheric

Environment, v.42, n.14, p.3351-3363, 2008.

MUNIZ, E.C.; FASCINA, V.B.; PIRES, P.P.; CARRIJO, A.S.; GUIMARÃES, E.B. Histomorphology of bursa of Fabricius: effects of stock densities on commercial broilers.

Revista Brasileira de Ciência Avícola, v.8, n.4, p.217- 220, 2006.

NAGARAJ, M.; WILSON, C.A.P.; SAENMAHAYAK, B.; HESS, J. B.; BILGILI, S. F. Efficacy of a litter amendment to reduce pododermatitis in broiler chickens. Journal of Applied Poultry Research, v.16, p.255-261, 2007.

NATIONAL CHICKEN COUNCIL NCC. National Chicken Council Animal Welfare Guidelines and Audit Checklist. 2010. Disponível em: $<\mathrm{http}$ ://www.nationalchickencouncil.co $\mathrm{m} /$ aboutIndustry/detail.cfm?id=19>. Acesso em: 12 abr. 2016.

OLIVEIRA, A.F.G.; BRUNO, L.D.G.; MARTINS, E.N.; ELIS, E.R.M.; MONTEIRO, A.C.; LEITE, M.C.P.; POZZA, P.C.; SANGALI, C.P. Efeito da densidade de criação e do grupo genético sobre a composição mineral e desenvolvimento de ossos longos de frangos de corte. Ciências Agrárias, v.35, n.2, p.1023-1034, 2014.

OLIVEIRA. M.C.; GOULART. R.B.; SILVA. J.C.N. Efeito de duas densidades e dois tipos de cama sobre a umidade da cama e a incidência de lesões na carcaça de frango de corte. Ciência Animal Brasileira, v.3, n.2, p.7-12, 2002. 
SANTOS, R.L.; NUNES, V.A.;

BAIÃO, N.C. Pododermatite de contato em frangos de corte. Arquivo

Brasileiro de Medicina Veterinária e Zootecnia, v.54, n.6, p.655-658, 2002.

SANOTRA, G.S.; DAMKJER LUND, J.; VESTERGAARD, K.S. Influence of light-dark schedules and stocking density on behaviour, risk of leg problems and occurrence of chronic fear in broilers. British Poultry Science, v.43, n.3, p.344-354, 2002.

SHEPHERD, E.M.; FAIRCHILD, B.D. Footpad dermatitis in poultry. Poultry Science, v.89, n.10, p.2043-2051, 2010.

SOUZA, A.P.O.; MOLENTO, C.F.M. Good agricultural practices in broiler chicken production in the state of Paraná: focus on animal welfare. Ciencia Rural, v.45, n.12, p.22392244, 2015.

TURRA, F. Avicultura e suinocultura do Brasil: produção e Exportação; Previsões para 2015 e 2016. Disponível em: $<$ http://abpabr.com.br/noticia/producao-de-carnede-frango-totaliza-13146-milhoes-detoneladas-em-2015-1545>. Acesso em: 12 abr. 2016.

WEBSTER, A.B. Welfare implications of avian osteoporosis. Poultry Science, v.83, n.2, p.184-192, 2004.

Data de recebimento: $24 / 05 / 2016$

Data de aprovação: 10/08/2016 